\title{
REDUÇÃO DE RISCOS DE DESASTRES. AS CONFERÊNCIAS REGIONAIS DA UNISDR EM 2014 E AS PERSPECTIVAS DE COOPERAÇÃO INTERNACIONAL EM CIÊNCIAS E TECNOLOGIAS.
}

\author{
REDUCCIÓN DEL RIESGO DE DESASTRES. CONFERENCIAS REGIONALES \\ DEL UNISDR EN 2014 Y LAS PERSPECTIVAS DE LA COOPERACIÓN \\ INTERNACIONAL EN CIENCIA Y TECNOLOGÍA.
}

\section{DISASTER RISK REDUCTION. UNISDR REGIONAL CONFERENCES IN 2014 AND THE PROSPECTS FOR INTERNATIONAL COOPERATION IN SCIENCE AND TECHNOLOGY.}

\author{
Ronaldo Lyrio BORGO ${ }^{1}$ \\ Darly Henriques da SILVA ${ }^{2}$
}

\begin{abstract}
RESUMO: O artigo comenta posicionamentos de atores governamentais em torno de políticas de Redução de Risco de Desastres e a orientação para elaboração de um framework global pós-2015 (HFA-02). Destaca como referências principais as difusões de Conferências das partes integrantes das Plataformas Regionais para a Redução de Riscos de Desastres como a das Américas e da Ásia, dentro do contexto da Estratégia Internacional de Redução de Desastres das Nações Unidas-UNISDR e do Programa de Ação Hyogo Framework for Action (HFA) de 2005 a 2015. Aspectos de avaliação de perdas e danos de desastres sobre a economia são abordados com base em documentação recente da UNISDR. São apresentadas sugestões acerca das possibilidades de cooperação multilateral regional entre a Ásia e Américas, em ciências e tecnologias aplicadas à gestão e redução de desastres, para compor o framework pós-2015 da UNISDR.
\end{abstract}

Palavras Chave: Desastres, Gestão e Redução de Riscos, Ações e Programas de Cooperação Internacional, Conferências e Plataformas regionais, Ciência e tecnologia.

RESUMEN: El artículo comenta las colocaciones de las políticas gubernamentales acerca de la Reducción del Riesgo de Desastres y la orientación de un marco global post-2015 (HFA02). Basado en las emisiones de las conferencias de las partes integrantes de la Plataforma Regional para la Reducción de Riesgo de Desastres de las Américas y de Asia, en el contexto de la Estrategia Internacional para la Reducción de Desastres (EIRD), y del Programa de Acción de las Naciones Unidas en el Marco de Acción de Hyogo e el periodo 2005-2015.

$1 \mathrm{MsC}$ Engenharia Elétrica-Telecomunicações - Satélite Emergenciais Aplicadas ao Brasil e AS. Analista Sênior de C\&T\&I no CNPq- Conselho Nacional de Desenvolvimento Científico e Tecnológico. E-mail: rlbaquarius@gmail.com

2 PhD Economia, Coordenadora Geral de Meteorologia, Climatologia e Hidrologia do MCTI ( 2004-2012). Analista Sênior de C\&T\&I no CNPq. E-mail: darlyhs@ @otmail.com 
Evaluación de las pérdidas por desastres y los impactos sobre la economía se abordan sobre la base de nueva documentación de la UNISDR. Sugerencias elementales se presentan sobre las posibilidades de cooperación regional multilateral entre Asia y las Américas, en las ciencias y las tecnologías para la gestión y reducción de los desastres, para componer el marco UNISDR posterior a 2015 .

Palabras clave: Gestión de Desastres y Reducción de Riesgos, Conferencias y plataformas regionales, Acciones y Programas de Cooperación Internacional, Ciencias y tecnologias.

SUMMARY: The article comments placements of governmental policies around Risk Reduction and Disaster guidance for a global post-2015 Framework (HFA-02). It's based on broadcasts from conferences of the integral parts of the Regional Platform for Risk Reduction Disaster as the Americas and Asia, within the context of the International Strategy for Disaster Reduction (UNISDR), the UN Programme of Action from the Hyogo Framework for Action (HFA) 2005-2015. Evaluation on the disaster losses and impacts on the economy are addressed based on fresh documentation of UNISDR. Elemental suggestions are presented about the possibilities of multilateral regional cooperation between Asia and the Americas, in science and technologies for management and disaster reduction, to compose the framework post-2015 UNISDR.

Keywords: Disaster Management and Risk Reduction, International Cooperation Programs, Conferences and regional platforms, Science and technology.

\section{Introdução}

O Risco de desastre é resultante de ameaças de eventos extremos e perigosos (hazards) ${ }^{3}$, da exposição, e das vulnerabilidades ${ }^{4}$ ambientais e sócio-econômicas das populações [9]. Os países desenvolveram ao longo do tempo seus métodos de gerenciar os riscos de desastres, e mitigar as vulnerabilidades, reduzindo-os através da construção de resiliências ${ }^{5}$. Criaram os meios para promover capacitações diversas para adaptação, com amortecimento e redução dos impactos, assim como fortalecer a capacidade de resistir e se recuperar de perdas e danos na

\footnotetext{
3 - Evento extremo e perigoso (hazard) é definido como: "um evento físico potencialmente prejudicial, fenômeno natural ou atividade humana que pode causar a perda de vidas ou ferimentos, danos à propriedade, ruptura social e econômica ou degradação ambiental. Riscos podem incluir condições latentes que podem representar ameaças futuras e pode ter diferentes origens: naturais (geológicas, hidrometeorológica e biológica) ou induzida por processos humanos (degradação ambiental e riscos tecnológicos). UNISDR. Genebra,2004.’[9](tradução do autor)

4 - A vulnerabilidade é definida como: "As condições determinadas por fatores ou processos físicos, sociais, econômicos e ambientais, que aumentam a susceptibilidade de uma comunidade ao impacto de Eventos extremos e perigosos( Hazards). UNISDR. Genebra, 2004.’[9] (tradução do autor)

5. "Capacitações sócio-econômicas e ambientais para minimizar os riscos e os impactos de desastres naturais e antropogênicos: capacitações em recursos humanos e materiais, infraestruturas e tecnologias disponibilizadas na prevenção de riscos emergenciais, na preparação de amortecimento, atenuação e mitigação de efeitos de variações climáticas bruscas e impactos de desastres."[21]
} 
ocorrência de desastres ${ }^{6}$. Há indutores de risco subjacentes, como o desenvolvimento econômico desigual, o crescimento urbano e regional mal planejado com expansão desordenada, o declínio de regulação dos serviços ambientais, a pobreza e desigualdade, a má governabilidade das capacidades locais, e mudanças climáticas, que compõem o risco de desastres. Estes também condicionam a capacidade de resistência aos impactos (resiliências) das famílias, comunidades, empresas e setor público. Desta forma, estes fatores influênciam a escalada de ocorrência de desastres e produzem impactos no curto e longo prazos. Embora o risco de desastres seja um problema enfrentado por todos os países, o risco tende a ser maior e a resistência menor em países pobres e em desenvolvimento. [9,19]

\section{Antecedentes}

O tema central do artigo "Redução de Riscos de desastres" é controverso e fez história na transição dos séculos XX p $\backslash$ XXI, como documenta o parágrafo dos "Elementos sugeridos pelo Comitê Preparatório da $3^{\text {a }}$ Conferencia Mundial das Nações Unidas para o framework sobre redução de desastres pós-2015” [20]:

“1. O Plano Estrutural de Ação lançado em 1989, sobre a Década Internacional para a Redução de Desastres Naturais, a estratégia de Yokohama de 1994 para um mundo mais seguro, e a Estratégia Internacional da ONU de 1999, para Redução de Desastres, formam a base para a Estratégia pós-2015 para a redução de risco de desastres".[20] (tradução do autor)

\section{Atualidades no Front da UNISDR - 2014:}

A geração de resiliências, tem no fortalecimento da Cooperação Internacional uma diretriz indicada pelo Comitê preparatório para a $3^{\mathrm{a}}$ Conferência Mundial da UNISDR pós2015 ou HFA-02, com ênfase no desenvolvimento de estratégias regionais e na criação de plataformas regionais:

"2. A adoção e implementação do Plano Estrutural de Acção de Hyogo para o período2005-2015: Construir Resiliência a Desastres das Nações e Comunidades, lançou um marco para catalisar os esforços nacionais e locais para reduzir o risco de desastres, e para o fortalecimento da cooperação internacional através do desenvolvimento de estratégias regionais, planos e políticas, e a criação de plataformas globais e regionais para a redução do risco de desastres, bem como a adoção do Plano de Acção da ONU para a Redução de Risco de desastres através de geração de Resiliência, pelo sistema das Nações Unidas".[20]( tradução e grifos do autor)

\footnotetext{
${ }^{6}$ _ "Os desastres são resultado de eventos adversos, naturais ou provocados pelo homem, sobre um ecossistema vulnerável, causando danos humanos, materiais e ambientais, e consequentes prejuízos econômicos e sociais". (Política Nacional de Defesa Civil (2007), p.8)
} 
O posicionamento do Comitê responde a uma preocupação maior da comunidade internacional e da ONU na medida em que afeta direta ou indiretamente todos os países, e faz um chamado à cooperação internacional entre as nações e regiões do planeta.

A Conferência Asiática para a Redução de desastres (ACDR) (em Tóquio/Japão de 4 a 6 de março de 2014) e a IV sessão da Plataforma Regional para a Redução de Riscos de Desastres das Américas (em Guayaquil/Ecuador de 27 a 29 de maio de 2014), emitiram contribuições regionais acerca de políticas intergovernamentais para compor um novo framework global pós 2015, ou Hyogo Framework for Action - HFA 02 para a redução de desastres. Tais eventos aconteceram um quarto de século após a Assembléia das Nações Unidas ter declarado ser a década 1990-2000, o decênio Internacional para a Redução de Desastres Naturais. Provavelmente, e como consequiência da $3^{\mathrm{a}}$. Conferência Mundial sobre Redução de Riscos de Desastres (WCDRR-2015) prevista para ocorrer em Sendai no Japão de 14 a 18 de março de 2015, deverá surgir uma nova Conferência das Partes, a COP mundial para a Redução de Desastres, que deverá incorporar os maiores esforços mundiais para "salvar vidas, recursos e meios de subsistência". [1,2]

A escalada crescente de perdas por desastres [14] e a corrida para a redução de desastres [9], fundam uma nova odisséia da humanidade acerca da Adaptação no século XXI. Desafia completamente a retórica política e sociológica alicerçada sobre a velha academia paradigmática do desenvolvimento sustentável e da globalização de mercados. Desafia governos e blocos econômicos, blocos políticos e uniões de nações, em torno de geração de reiliências para adaptação com a redução de riscos de desastres na busca austera por sustentabilidade. A Figura 1 ilustra o comportamento dos desastres naturais, com índices de 2012.[15]

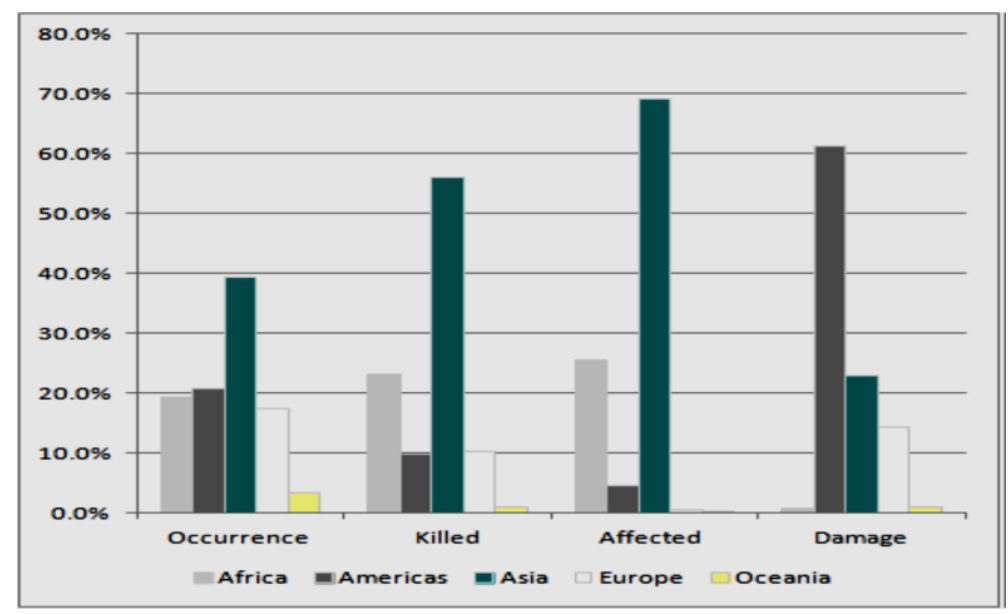

Figure 1: Impacts of Natural Disasters by Region, 2012. Source: EM-DAT: The OFDA/CRED International

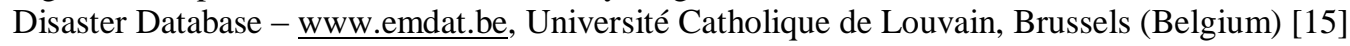

A Ásia lidera a ocorrência, as mortes e os afetados pelos desastres, porém, nas Américas que os danos materiais se mostram mais devastadores, ou seja onde ocorrem os maiores índices de perdas e danos traduzidos em prejuízos econômicos. Considerando que a América do Norte tem seus frameworks bem estabelecidos para a gestão e redução de riscos de desastres[16], ainda assim quanto aos danos econômicos, as Américas marcaram 61,1 por cento de participação, devido principalmente a furacões e tempestades que devastaram os Estados Unidos em 2012[15]. Ademais, as Américas situam-se em $2^{\circ}$. lugar no ranking de ocorrências de desastres hidrometeorológicos, sendo que na região América Latina e Caribe faltam 
frameworks integrados para Gestão e Redução de desastres, assim como para Adaptação às Mudanças Climáticas.[7,18]

O Relatório Global de Avaliação da Redução de Riscos de Desastres da UNISDR (GAR 2013) adverte em sua apresentação "GAR at a Glance: The Wake-up call: Disaster are costlier tham we though" e apresenta balanços econômicos dramáticos sobre as realidades conjunturais globais no novo século[8]:

"A maioria dos desastres que poderiam ocorrer ainda não aconteceram: O Total esperado de perda anual global equivalente aos danos por ocorrência de terremotos e ciclones atinge o equivalente a cento e oitenta bilhões de dólares (US\$ 180.000.000.000) por ano (Capítulo 3). Este valor não inclui o custo significativo de desastres locais por ocorrêcia de inundações, deslizamentos de terra, incêndios e tempestades (Capítulo 4) ou o custo de interrupção de negócios. A agricultura também está em risco: em Moçambique uma seca ocorrente para cada um em dez anos(01/10), reduziria a produção de milho em 6 por cento e 0,3\% do PIB (Capítulo 6). " $[8]($ Tradução do autor);

I) Referendando a citação acima do GAR 2013, as figuras 3 e 4, com dados agregados e globais sobre os desastres, apontam os danos econômicos globais de 1983 a 2012, tendo sido mais devastador economicamente de 2008 a 2012, com maior ocorrência entre 1998 e 2002[15]:
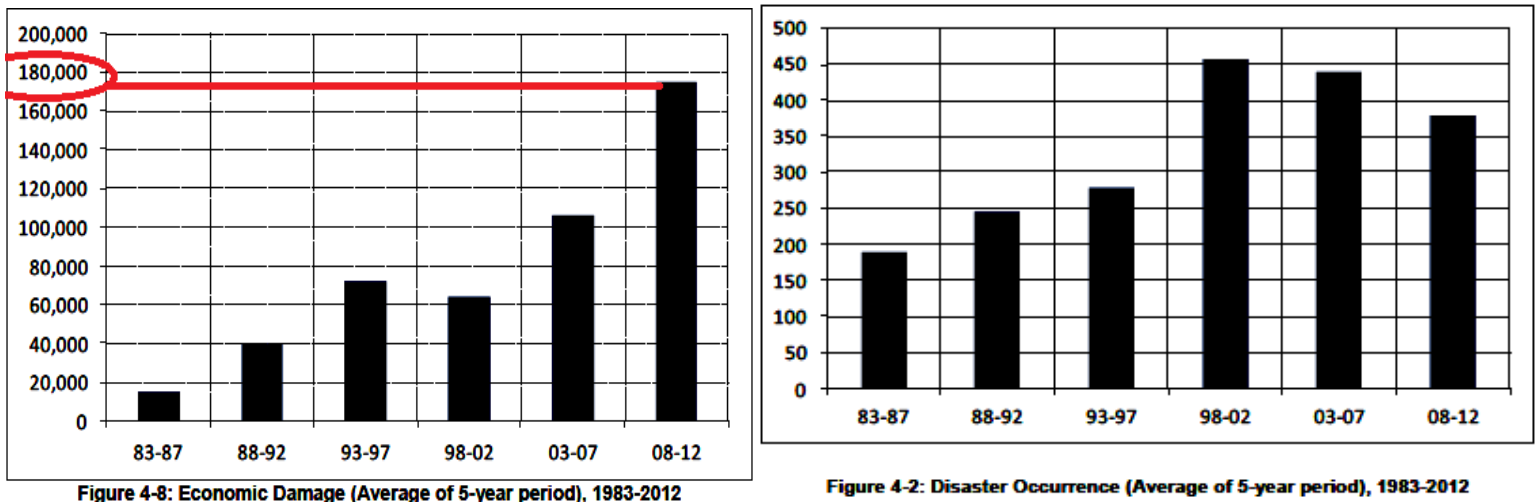

Figura 3 e 4- Perdas e Danos Econômicos e ocorrências de desastres no período 1983 a 2012. [15]

II) O Relatório GAR 2013 faz um balanço marcante dos desastres ocasionados pelas queimadas das florestas tropicais e do impacto relativo a perdas de capital natural que sustenta a riqueza da humanidade, e reflete o risco compartilhado globalmente nos valores das perdas potenciais de ecossistemas tropicais [8]:

"Riscos incidentes sobre o Capital Natural comprometem a saúde econômica do futuro: Riscos de desastres incluem perdas e danos ao capital natural com graves consequências para as empresas, as famílias e riqueza de um país. Por exemplo, incêndios florestais incontrolados agora afetam todos os continentes, com perdas globais anuais sobre os ecossistemas tropicais potencialmente atingindo cento $e$ noventa bilhões de dólares (US\$190.000.000.000) (Capítulo 6). A degradação do solo aumenta o risco de seca agrícola; na África, a área total com alta degradação e 
alto risco de seca é de quase 260 mil quilômetros quadrados” [8](Tradução do autor);

III) O Relatório dispara uma campanha de marketing em torno da idéia de que a Gestão de Risco de Desastres se tornará alvo de maior foco entre os "business cases" dos maiores líderes da economia internacional [8]:

"Não é mais"business as usual": Grandes catástrofes recentes, como o furacão Sandy em 2012 e as inundações na Tailândia em 2011, chamaram a atenção para o crescente impacto dos desastres no setor privado (Capítulo 1). Muitas das grandes empresas globais estão agora fortalecendo sua capacidade de gestão de risco. No entanto, as empresas ainda apresentam um "foco-cego" para o risco de desastres, que é amplamente ignorado nas previsões econômicas e projeções de crescimento (Capítulo 12). "[8](Tradução do autor);

"À medida que nos aproximamos de 2015, os esforços internacionais estão intensificando a formulação de um novo cenário para a redução de risco de desastres. A garantia de que o "business case" para a redução do risco de desastres é explicitamente incluída nesse cenário fornecerá um incentivo fundamental para o engajamento construtivo, por parte das empresas, em que resiliência, competitividade e sustentabilidade de futuro dependem." [8](Tradução do autor);

\section{Cenários, Posicionamentos e Perspectivas da UNISDR-Las Américas}

As recomendações da Conferência de Guayaquil refletem as experiências consolidadas em praticamente meio século, em políticas de gestão de risco de desastres como a CentroAmericana e do Caribe, que redundou na Comprehensive Disaster Management Strategy - CDM(2014 a 2024), e que são amparadas em mecanismos de integração e cooperação regionais como CARICOM, SICA, UNASUL, AEC, CELAC e OEA.[1] Destacam-se os itens das Recomendações de Guayaquil que aderem completamente às da Sessão 2 - "Strengthening Local Capacity for DRR" do Relatório síntese da Conferência Asiática sobre a Redução de Desastres (março 2014, Tóquio/Japão)[2], e com o Posicionamento das Sociedades Civis[5]. O consenso de idéias baseadas nas experiências e no entendimento acerca da necessidade de implementar atividades com eficácia e eficiência para a redução de riscos de desastres, ou seja, a geração de resiliências sócio-econômicas e ambientais, está intensivamente baseado na promoção de mecanismos de fomento às iniciativas locais de governança para a redução de riscos de desastres $\operatorname{RRD}[1,2,5]$ :

"Criar mecanismos e instrumentos que permitam aos governos locais terem acesso a distintos tipos de recursos e à cooperação científica, técnica e financeira de forma descentralizada"; "Fomentar a associatividade, as redes, as comunidades rurais, as alianças das cidades, assim como a participação cidadã da sociedade civil $e$ comunidades locais, para alcançar governança local, nacional, regional $e$ internacional de risco e resiliência urbana". [5](Tradução do autor)

As recomendações de Guayaquil dão ênfase à importância das culturas e de saberes locais que remetem ao conhecimento dos povos tradicionais e indígenas, acerca da geração de resiliências para promoção de Adaptação aos eventos extremos do tempo e clima, além da 
insofismável valorização das comunidades locais no trato da gestão de riscos e na própria gestão de desastres.[5]

Em todas as declarações de representantes da diplomacia governamental dos países Americanos nota-se a importância de enfatizar as diretrizes nacionais para garantir efetividade das ações e programas que suportam a gestão de riscos de desastres[1]. Na declaração dos EUA estão demarcados aconselhamentos retóricos e repetitivos para os demais países, considerando-se que têm implementado um programa de alto custo operacional em cobertura nacional a partir de 2011, The National Mitigation Framework. Este incorpora a redução de desastres como prioridade, e dispõe de orçamento de Estado atribuído (The National Predisaster Mitigation (PDM) Fund) no contexto dos trinta e cinco anos de uma Agência Federal de Gestão de Emergências (FEMA). Esta instituição é dedicada e com cobertura nacional à gestão e redução e desastres, incorporada ao Departamento de Estado para a Segurança Territorial dos EUA (US Homeland Security Department)[16]:

"Infelizmente o padrão global de extremos do clima e as devastações têm se tornado rotineiras, e os governos devem se preparar para ajustar suas políticas e práticas para considerar e redirecionar recursos para gerenciar riscos de forma a minimizar as devastações. Para sermos exatos, os recursos para enfrentar estes riscos são limitados tanto quanto a maioria dos países enfrentam desafios e problemas econômicos muito complexos que não terão soluções de curto prazo. Consequentemente, os esforços para a redução de desastres (Disaster Risk ReductionDDR) devem ser inteligentes e dinâmicos, acessíveis ao nível local, e destinados a atingir resultados práticos. Ações de preparação robustas em todos os níveis podem alcançar e prevenir significativamente as destruições e perdas de vida." [4] (Tradução do autor)

Dentre as diversas declarações dos países membros da UNISDR - Las Américas, destacam-se vetores orientados para o HFA2, ou Framework pós-2015 para a Redução de desastres, porém sem qualquer posicionamento quanto a estratégias de cooperação internacional entre as regiões Norte-Sul e Sul-Sul inovadoras em Ciência e Tecnologia, além da vertente política ambientalista que pretende promover planos(frameworks) voltados para empreendimento da "green-economy" em escalas regionais, como forma indireta de reduzir riscos de desastres naturais construindo cidades mais resilientes aos extremos climáticos, e mudando paradigmas de produção e consumo energético do tipo "CO2 free".

Destaca-se nos posicionamentos da UNASUL grandes preocupações voltadas para o estabelecimento de uma forte cultura de redução de riscos de desastres(RRD) nos países membros, baseada em diretrizes bem formuladas, como: a criação de capacidades de intercâmbio de experiências à nível regional e local; a promoção de aliança/acordo públicoprivados em gestão de riscos de desastres (GRD)[1,2]. No entanto, nota-se, nas diversas declarações dos países membros da ALC, muita retórica acerca de políticas públicas em torno de planejamento de ações normativas para induzir os governos a adotar os posicionamentos e estratégias apontadas nas "Recomendaciones para el Marco Internacional post 2015 para La Reduccíon del Risco de Desastres (HFA 2)". A posição mais acertada está postulada pelo grupo tecnico-científico[4] que reuniu os representantes das academias da região, com larga experiência e visão estratégico-científica junto às inúmeras iniciativas de gestão e redução de desastres e que expressa a seguinte opinião acerca do assunto: 
"Reconhecemos que os objetivos de desenvolvimento sustentável somente serão alcançados se a Redução de Riscos de Desastres e a Adaptação às Mudanças Climáticas forem incorporadas a um plano integrado de desenvolvimento [4](Tradução do autor)

De forma geral, a UNISDR-Las Américas deixa transparecer que as iniciativas regionais para a gestão e redução de desastres ainda não frutificaram. Mesmo existindo iniciativas nacionais ainda centralizadas e em fase de pilotos locais/intra-regionais, elas exibem pouco alcance e cobertura nacionais, com baixa eficácia e eficiência na redução de riscos de desastres. $\mathrm{O}$ excerto da avaliação realizada no Brasil pertinente à implementação do HFA no período 2011 a 2013, revela pontos fracos e a busca por cooperação internacional no âmbito da UNASUL[6]:

"Infelizmente a parceria do Governo Brasileiro com os outros países da América do Sul ainda não está consolidada e caminha a passos lentos, parte por falta de iniciativa do Governo Brasileiro, parte por iniciativa dos países vizinhos. Algumas tímidas cooperações internacionais em matéria de Defesa Civil estão sendo iniciadas, mas infelizmente, não têm uma continuidade sistemática com o passar dos anos."

"A realização de Plataformas Regionais e outros eventos de Defesa Civil, com o importante encorajamento da Estratégia Internacional para a Redução de Desastres(EIRD) tem contribuído para mudar este quadro, porém sem grandes resultados concretos pelo momento. Apesar de possuir uma das maiores fronteiras terrestres do planeta, mais de $95 \%$ da população brasileira mora afastada das fronteiras, se concentrando em grandes centros urbanos próximos do litoral. Devido a isso, há pouca atenção em matéria de Defesa Civil do Governo Federal em zonas fronteiriças. A maior parte das cooperações internacionais entre o Governo Federal e outros países da América do Sul concentra-se na troca de técnico e experiências em matéria da prevenção e redução de riscos em grandes centros urbanos, foco da Defesa Civil Nacional." [6]

De fato, falta vontade política para cooperar. Seja com base em integração de plataformas tecnológicas e infraestruturas para gestão e redução de desastres, seja na base de repartição de investimentos e disponibilização de fundos de desenvolvimento por parte dos grupos de países ricos como EUA, Canadá, UE e Japão. Isto também ocorre no contexto dos blocos e Uniões como a UNASUL, já que ciências e tecnologias estão disponíveis e experiências diversas estão implementadas principalmente nos países ricos e na região AsiaPacifico [7]. É positivo o argumento de se queimar etapas absorvendo as técnicas e metodologias mais exitosas em gestão de riscos e redução de desastres. Mas também, via mecanismos e acordos de cooperação internacional, frente à escalada de perdas econômicas e vitais que se apresentam neste início de século XXI, onde estão bem caracterizadas as demandas por Adaptação às Mudanças Climáticas e de Redução de Riscos de Desastres. Tais demandas emanam dos processos da própria globalização de mercados e da corrida dos blocos econômicos regionais, tratadas, insofismavelmente, como assuntos de Estado pelos países e blocos de maior tradição em governança para o desenvolvimento. Porém, entre os blocos remanescentes da UNISDR-Las Américas, faltam entendimentos para encarar estes assuntos como prioritários, para que seus orçamentos anuais não sejam batidos pelos prejuízos dos desastres e catástrofes naturais. Avaliações das perdas econômicas devido a desastres realizadas já na $2^{\text {a }}$ década do séc. XXI, confirmam categoricamente estas afirmações extra-oficiais, como mostrado na Figura 6. Os países desenvolvidos perderam cerca de $11 \%$ 
do PIB acumulados nas duas décadas que seguiram a transição dos séculos XX para XXI, os países em desenvolvimento cerca de $14 \%$, os subdesenvolvidos perderam até $127 \%$ e os Estados-ilhas até $365 \%$ dos seus respectivos PIBs. [14]

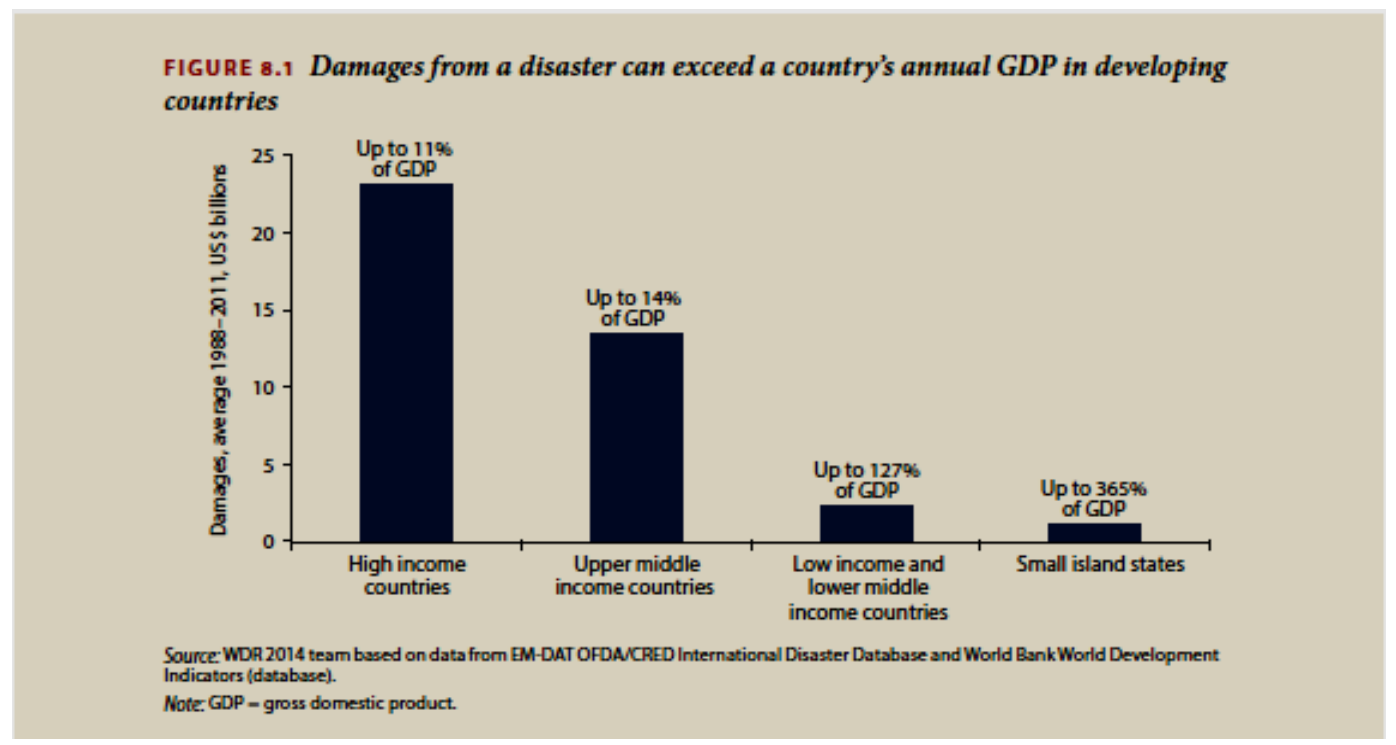

Figura 6- Fonte: WDR 2014[14]

\section{Evidências de Escalada de Contra-Economia por Desastres}

Desde a Cúpula do Clima ocorrida em Cancún/México durante a COP-16 em 2010, o relatório Economia do Clima na América Latina (ECLAC) já destacava, com ênfase no balanço de perdas e danos havidos, e sobre as projeções de mudanças climáticas entre 2010 e 2100, em torno de $1 \%$ do PIB a escalada de perdas e danos em decorrência de desastres, supostamente condicionados pelas ações da UNFCCC no período, para mitigação de $\mathrm{CO} 2[7]$.

\begin{tabular}{|c|c|c|c|c|}
\hline \multirow[b]{3}{*}{ ANO } & \multirow{2}{*}{\multicolumn{2}{|c|}{$\begin{array}{l}\text { PIB } \\
\text { (trilhões R\$) }\end{array}$}} & \multicolumn{2}{|c|}{ ESTIMATIVA PERDAS DESASTRES } \\
\hline & & & ECLAC $\quad 1 \% \mathrm{PIB}$ & \\
\hline & ALC & & ALC & BRASIL \\
\hline 2011 & 5,66 & 4,1 & 56,6 & 41 \\
\hline 2012 & 6 & 4,5 & 60 & 45 \\
\hline 2013 & 6,4 & 4,84 & 64 & 48,4 \\
\hline
\end{tabular}

Quadro I: Estimativas de perdas econômicas por desastres com base na ECLAC/COP-16.

Este fator de escala em perdas representa somente os prejuízos em infraestrutura e bens econômicos, e não avalia as perdas de vidas humanas para a sociedade. Tais montantes relacionados com perdas e danos decorrentes de desastres superam orçamentos dos Estados da região da ALC, comprometendo todos os esforços de investimentos em desenvolvimento, integração e sustentabilidade. Tomando-se os valores do PIB de 2011 a 2013, estima-se em dezenas de bilhões de dólares/ano para perdas econômicas por desastres para o Brasil e região 
da ALC. Em termos comparativos, estes valores cobririam orçamentos destinados aos investimentos totais em frameworks para gestão e redução de desastres, como o "SENTINEL ASIA" que abrange dezenas de países da região Ásia-Pacífico [13,22], ou ainda, os frameworks conjuntos da FEMA que cobrem os Estados Unidos da América do Norte no século XXI [16].

Este é um tema de economia reversa, ou de contra-economia, que enfatiza a relação custo-benefício pertinente à geração de resiliências, para a redução de riscos de desastres, e que merece ser adotado diante das realidades postas pelas evidências de mudanças climáticas globais. As parcerias são um instrumento indicado para minimizar os custos e otimizar os resultados. As contingências por riscos de desastres no século XXI, impõem posicionamentos claros por parte dos Estados-Nações e dos respectivos blocos regionais em torno de desafios de cooperação em novas bases políticas e técnico-científicas para alcançar índices prováveis e aceitáveis de Adaptação no médio e longo prazos[18].

\section{Acerca do Posicionamentos da Conferência Asiática para a Redução de desastres (ACDR)}

Considerando um acúmulo de experiências em aproximadamente meio século em gestão de desastres e adaptação a fenômemos extremos hidrometeorológicos, geológicos e biológicos, adquiridas por necessidade de proteção contra esses fenômenos, os países da região Àsia-Pacífico desenvolveram plataformas tecnológicas integradas proprietárias como a "SENTINEL ÁSIA"[13,22]. Criaram também associações de iniciativas de âmbito regional como a Asian Disaster Reduction Center (ADRC)[12], além de se associar com o Consórcio Internacional CHARTER Space and major disasters, estabelecendo backbones em plataformas espaciais alternativas e contingentes $\mathrm{p} \backslash$ a prevenção e gestão de riscos de desastres. A iniciativa "SENTINEL ASIA" estabeleceu uma Plataforma tecnológica espacial e terrestre para suportar o ciclo completo de gestão e redução de desastres operacionalmente neste século[11]. A iniciativa resultou de um amplo acordo de cooperação entre as Agências Espaciais e Agências de Gestão de desastres dos países da Região Ásia-Pacífico, a AsiaPacific Regional Space Agency Forum (APRSAF). A iniciativa "SENTINEL ASIA" conta ainda com atuação integral do Asia-Pacific Center for Technology Transfer (APCTT) e do Asia-Pacific Trainning Center for Information and Communication Technology for development (APCICT). Este framework que se encontra em sua $3^{\mathrm{a}}$ fase de expansão, é o maior exemplo de União Política em bloco regional para a integração de infra-estrutura de ciências e tecnologias, e de cooperação internacional multilateral para suporte pleno à gestão de riscos de desastres (DRM) e redução de riscos de desastres (DRR). Sua importância estratégica reside no fato que a plataforma tecnológica é compartilhada por todos os países da região, promovendo capacitação, seja de recursos humanos, como de infra-estruturas de sistemas e plataformas tecnológicas de monitoramento, controle, processamento, comunicação e informação plena para gestão e redução de desastres [22]. Esta soma de experiências e conhecimentos estão destacadas nas atas da Conferência Asiática UNISDR2014, em sua Sessão 4- Utilização de tecnologia espacial para a Redução de Risco de Desastres (Session 4: Utilization of Space Technology for DRR)[2], que enfatizam os seguintes meios e para atingir os objetivos:

"Melhorar a utilização de imagens de satélite para a redução de riscos de desastres (DRR): As apresentações da Asian Institute of Technology (AIT), Agência Japonesa de Exploração Aeroespacial (JAXA), o Instituto Filipino de Vulcanologia e Sismologia 
(PHIVOLCS), o Centro Asiático para redução de desastres (Asia Disaster reduction Center-ADRC), e do Ministério da Administração de Desastres e Socorro de Bangladesh, mostraram como as tecnologias espaciais têm contribuído para reforçar os esforços DRR na região e tornando-se parte integrante dos sistemas de DRM nacionais. A utilização de imagens de satélite integra os sistemas de alerta precoce quanto à identificação, avaliação e monitoramento dos riscos, fornecendo capacitações únicas. A conferência recomendou que as perspectivas da mais ampla utilização dessas tecnologias devem ser exploradas para beneficiar mais países membros da ADRC, especificamente através do framework "Sentinel Ásia". Recomenda-se também que o uso de tecnologia espacial seja integrado no HFA2."( Tradução do autor)

\section{Conclusões e Recomendações acerca do HFA post- 2015.}

Como resultados das compilações das consultas feitas sobre o framework pós 2015 para a redução de desastres em nível internacional, após decorridas as conferências regionais[17], cabem diversas questões listadas a seguir:

1-O que os governos querem e podem realizar em termos de políticas de ciência e tecnologias para atender as demandas impostas pelos desastres, e o que realmente importa para o ambiente mundial?

2 - Como canalizar recursos para a redução de desastres onde nem sequer existem infraestruturas de saneamento básico e urbanização resiliente? Os países desenvolvidos estão dispostos a colaborar com os em desenvolvimento, quando já são onerados por outras atividades, como as "humanitárias", por exemplo, que não são poucas dadas às guerras tradicionais e os campos de refugiados patrocinados por órgãos internacionais?

3- Não estaremos percutindo velhas fórmulas com novos rótulos programáticos para assentar mecanismos de desenvolvimento "sustentável" baseados em velhos paradigmas de desenvolvimento do gênero " business as usual”?

4- Por que sistematizar metodologias de cooperação multilateral tão somente no âmbito regional clássico, se atualmente há arranjos em blocos de interesses econômicos de desenvolvimento com vetores multilaterais entre as regiões Norte-Sul e Sul-Sul, como o BRICS (Brasil, Rússia,Índia e China) derivados de vários precursores como o IBAS( Índia, Brasil, África do Sul), sem desconsiderar os esforços dos blocos clássicos como CARICOM, ASEAN, e outros?

5- Como aplicar a economia reversa tendo em vista as realidades locais e regionais, embora o poder devastador dos desastres afetem sempre mais dramaticamente os países em desenvolvimento devido ao seu menor poder de reação?

As possibilidades de cooperação internacional e regional entre a Ásia e as Américas, são amplas na grande área de gestão e redução de riscos de desastres em dimensões continentais. Tanto a América do Norte quanto a Ásia têm trajetórias próprias, com 
aproximadamente meio século de experiências em gestão e redução de desastres com consolidação de frameworks regionais no século XXI, sem menosprezar os desenvolvimentos na Europa e Ásia-Pacífico, que se ergueram na base da cooperação técnico-científica.. Contam também com um amplo espectro de políticas e práticas de gestão operacional, de construção de capacidade tecnológica e humana para enfrentar o desafio de reduzir riscos e mitigar impactos, além da evolução da missão da defesa civil na prevenção e resposta a desastres.

Ao contrário das Américas Latina e do Sul, que embora tenham diversas iniciativas nacionais e uma gama interessante de organizações da sociedade civil empenhadas em mobilizar os governos e fazer políticas intergovernamentais de peso apropriado, ainda não alcançaram o desenvolvimento de relações internacionais para a promoção de ações intergovernamentais, tais como:

I-Políticas e programas de Estado, que subsistam aos governos e sejam delongo prazo;

II-Uniões e Associações de agências espaciais e agências/centros de gestão de desastres regionais dedicados aos problemas dos desastres naturais e antropogênicos;

III- Ações de médio e longo prazos em bloco de estados, importantes para países continentais, como o Brasil. Esta experiência pode ser angariada em cooperação internacional direcionada a ganhar tempo em salvar milhões de vidas, preservar desenvolvimentos e o que ainda resta de recursos naturais da biodiversidade.

A reunião da UNISDR em 2015 poderá facilitar e impulsionar acordos fundamentais, caso haja vontade política de superar a condição estrutural global, e, avançar com cooperações frutíferas para promover adaptação às mudanças climáticas e redução de riscos de desastres.

\section{Referências}

Asian Disaster Reduction Center. Natural Disaster Data Book 2012 (An Analytical Overview). ADRC 2012.

Biennial HFA Progress Review 2007-2009. Space Technology Applications for Disaster Risk Reduction. United Nations Economic and Social Commission for Asia and the Pacific (ESCAP).Asian Disaster Reduction Center (ADRC). 
BORGO, R. L. Iniciativa Regional na America Latina \& Caribe para Gestão e Redução de desastres no século XXI. Revista Terceiro Incluido - ISSN 2237-079X - NUPEAT-IESAUFG, v.2, n.2, jul./dez., 2012, p. 100-109, Artigo 31.

BORGO, R. L. Estudo de desempenho de sistema via satélite geoestacionário nas bandas Ka e $\mathrm{Ku}$ para suporte de telecomunicações a uma rede de gestão e redução de desastres. 2012. Dissertação de Mestrado. Universidade de Brasília. Faculdade de Tecnologia. Departamento de Engenharia Elétrica.

BORGO, R. L. About the extreme climate changes adaptation and disaster risks management and reduction in the Latin América ad Caribbean Region. Revista Terceiro Incluído ISSN 2237-079X NUPEAT-IESA-UFG, v.3, n.1, Jan./Jun., 2013, p. 37-46, Artigo 36.

Consideraciones de los representantes de los sectores académicos, técnicos y científicos que participaron en la Cuarta Sesión de la Plataforma Regional para la Reducción de Riesgos de Desastres en las Américas. (Guayaquil, Ecuador - 27-29 Mayo 2014).

Compilation report on consultations on the post-2015 framework for disaster risk reduction. Considerations on the post-2015 framework for disaster risk reduction. Third United Nations World Conference on Disaster Risk Reduction Preparatory Committee First session, Geneva, 14-15 July 2014.

Comunicado de Guayaquil, Ecuador. IV sesión Regional para La Reduccíon Del Riesgo de Desastres de las Américas- UNISDR. Invertir em RRD para proteger los avances Del desarrolo.Guayaquil, 29 mayo 2014.

Declaracíon Presidencia Grupo de Trabajo de Alto Nivel para La Gestíon Del riesgo de desastres de La Unión de Naciones Suramericanas (UNASUR).

DARLY, Henriques da Silva. Combate à Desertificação e a COP dos Pobres. Revista Terceiro Incluído ISSN 2237-079X NUPEAT-IESA-UFG, v.4, n.1, Jan./Jun., 2014.( No Prelo).

ESCAP Technical Paper.Collaborative Development of a Regional Disaster Management Support Platform on Space-based Information Sharing and Analysis. Information and Communications Technology and Disaster Risk Reduction Division. September 2009.

HFA Brazil. National progress report on the implementation of the Hyogo Framework for Action (2011-2013). Official report produced and published by the Government of 'Brazil'. http://www.preventionweb.net/english/countries/americas/bra/.

Joint Civil Society Position on Post 2015 Framework for Disaster Risk Reduction. FINAL GNDR, 3 June 2014.

KAKU, Kazuya. Alexander Held. Sentinel Asia: A space-based disaster management support system in the Asia-Pacific region. International Journal of Disaster Risk Reduction. 2013 The Authors. Published by Elsevier Ltd. All rights reserved.

KAWAI, M.; K. Kaku; S. Tanaka, A. Ono, T. Horiuchi. SENTINEL ASIA- International Cooperation for Disaster Management in the Asia-Pacific Region .Satellite Applications and Promotion Center, Japan Aerospace Exploration Agency, Tokyo, Japan. 
Summary Report. Asian Conference on Disaster Reduction 2014. UNISDR,ADRC. March 46,2014. Tokyo,Japan.

Third United Nations World Conference on Disaster Risk Reduction. Suggested elements for the post-2015 framework for disaster risk reduction. Preparatory Committee. Note by the secretariat. First session. Considerations on the post-2015 framework for disaster risk reduction. Geneva, 14-15 July 2014.

United Nations Economic and Social Commission for Asia and the Pacific (ESCAP). Policy Framework on Space Information Products and Services for Disaster Management: Study report synthesizing the recommendations of the Meeting of Experts on Policy Framework on Space Information Products and Services for Disaster Management. Beijing, 1. 2007.

U.S. Department of Homeland Security. Federal Emergency Management Agency National Pre-disaster Mitigation Program. Fiscal Year 2013 ,Congressional Justification. FEMA.

UNISDR (2013) From Shared Risk to Shared Value -The Business Case for Disaster Risk Reduction. Global Assessment Report on Disaster Risk Reduction. Geneva, Switzerland: United Nations Office for Disaster Risk Reduction (UNISDR).

World Conference on Disaster Reduction. Hyogo Framework for Action 2005-2015: ISD R. International Strategy for Disaster Reduction. Building the Resilience of Nations and Communities to Disasters. www.unisdr.org/wcdr. 18-22 January 2005, Kobe, Hyogo, Japan.

World Bank. 2013. World Development Report 2014: Risk and Opportunity-Managing Risk for Development. Washington, DC: World Bank. doi: 10.1596/978-0-8213-9903-3. License: Creative Commons Attribution CC BY 3.0. 